\title{
Was ist der Unterschied bei der Anzahl der Semester innerhalb eines Studienganges?
}

Inhaltlich unterscheiden sich die beiden Systeme erst mal nicht vom vermittelten Wissen. Der Unterschied liegt bei einem verpflichtenden Praktikum, das man bei einem siebensemestrigen Studiengang in der BWL absolvieren muss. Da Firmen auch für Praktikanten mittlerweile den Mindestlohn zahlen müssen, sind diese erpicht darauf, Pflichtpraktikanten einzustellen, da diese geringer bezahlt werden können [11]. Dadurch sind hier die Chancen größer, ein interessantes Praktikum zu ergattern. Der Nachteil für Studenten ist jedoch, nicht gerade viel für seine Arbeit zu bekommen. Letztlich ist ein Praktikum aber eine gute Vorstellung in einem Unternehmen und wenn man sich hier bewährt, kann es durchaus zu Jobangeboten und dergleichen kommen. Auch gewinnt man dadurch erste Berufserfahrung, die sich im Lebenslauf nie schlecht macht. Der Vorteil eines sechssemestrigen Studienganges ist, dass man ein halbes Jahr früher in die Arbeitswelt oder in den Master starten kann. Gerade wenn man bereits vor dem Studium Arbeitserfahrung im gewünschten Beruf gesammelt hat, ist dies attraktiv. Bei Unentschlossenen und Studenten ohne Arbeitserfahrung ist jedoch die siebensemestrige Option meist die bessere Wahl. Für mich als vorerst eher unentschlossenem Studenten hat es sich auf jeden Fall ausgezahlt, um mehr Gewissheit über meinen Berufswunsch zu erhalten. Wie sich die jeweiligen Studiengänge dann zusammensetzen, findet man immer im Curriculum oder der Studiengangbeschreibung.

An dieser Stelle wäre ein Beispiel für einen siebensemestrigen Studiengang mein eigener in Worms (International Business Administration and Foreign Trade Bachelor of Arts (B.A.) an der HS Worms).

(https://www.hs-worms.de/iba-bachelor/) [12]

Und ein Beispiel für einen BWL-Studiengang mit sechs Semestern ist International Business \& Economics (IBE) der Universität Augsburg.

(https://www.wiwi.uni-augsburg.de/ibe/studienverlauf/) [13] 